\title{
ESTADO NUTRICIONAL, RISCO DE SARCOPENIA E USO DE TERAPIA NUTRICIONAL EM IDOSOS HOSPITALIZADOS COM COVID-19
}

Juliana Heitich Brendler; Pontifícia Universidade Católica do Rio Grande do Sul; brendlerjuliana@gmail.com Anna Carolina Brum Portilho Martinez; Pontifícia Universidade Católica do Rio Grande do Sul; acarol_bpm@hotmail.com

\section{RESUMO}

Introdução: Conforme estudos sobre COVID-19, o vírus acomete de forma mais grave a população idosa, obesa e doente crônica. Além disso, seu diagnóstico foi associado à anorexia, perda ponderal e muscular, fatores fortemente associados à desnutrição e sarcopenia, que acarretam no aumento da morbimortalidade em idosos. Se torna essencial a avaliação nutricional e triagem de risco de sarcopenia nesse público, além da terapia nutricional adequada. Objetivo: Identificar dentre idosos internados com diagnóstico de COVID-19, o estado nutricional, risco de sarcopenia, bem como a intervenção nutricional aplicada. Métodos: Estudo observacional transversal, realizado em hospital terciário em agosto de 2020. Incluídos pacientes de ambos os sexos, maiores de 60 anos. Foram coletados, a partir do prontuário, dados antropométricos e utilização de terapia nutricional, e aplicou-se o SARC-F via telefone. As variáveis foram consideradas utilizando o percentual de concordância da amostra. Resultados: Foram identificados 17 idosos com COVID-19 na enfermaria, dos quais 88,2\% apresentaram risco para sarcopenia. Destes, $40 \%$ apresentaram baixo peso e $40 \%$ excesso de peso, conforme o IMC preconizado pela OPAS. Quanto à terapia nutricional, 33,3\% faziam uso de suplemento oral e $40 \%$ de via alternativa de alimentação com aporte hipercalórico/hiperprotéico. Conclusão: Em concordância com a literatura, foi possível identificar nesta amostra, grande número de idosos com risco de sarcopenia. Estes se encontram nos dois extremos da classificação do estado nutricional. Em relação a terapia nutricional, a maioria dos pacientes teve intervenção adequada, porém observamos que uma grande parcela dos que tinham dieta via oral poderiam ter uma otimização no aporte.

Palavras-chave: Sarcopenia; Idoso; Estado Nutricional; Infecções por Coronavirus. 